V. I. TIKHONOV, Dr. of Tech. Sciences, Prof. "ONAT", Odessa,

O. V. TYKHONOVA, teacher "ONAT", Odessa

\title{
COGNITIVE TENSOR MODEL OF A SYSTEM BASED ON VON NEUMANN CLASSES
}

The paper focuses issues of tensor calculus study in technical universities. To promote a holistic insight on tensors by undergraduates, there proposed a cognitive tensor model of a physical system on the base of hierarchical John Von Neumann classes. Refs.: 23 titles.

Keywords: cognitive tensor model; physical system; Neumann classes.

1. Introduction. The problem statement. System tensor modeling plays an important role in various fields of theoretical science and applied researches. The concept of tensor goes back to Riemann's ideas of nonEuclidean geometry on curved smooth surfaces presented in 1854 [1]. Tensor calculus itself was introduced by Ricci-Curbastro (Italy) in 1887 primarily as extension of vectors [2], and further developed by his student Levi-Civita ([3], 1899).

The tensor method of Ricci-Curbastro/Levi-Civita latter found its application in general relativity theory (GRT) coauthored by A. Einstein and M. Grossman in 1913 [4]. In subsequent decades, tensor analysis penetrated other areas, and now included in advanced academic courses at technical universities and engineering high schools [5 - 12]. Students often seem aware of tensor's importance, though, unless engaged in a dedicated course, tensor remains covered in a veil of mystery [10].

An adequate insight of tensor by undergraduate students meets two major issues: firstly, the lack in a clear presentation of tensors tied to what students already know from other courses, such as vector algebra; secondly, the use of cumbersome tensor notation and construction rules for high rank tensors [10].

In this work, typical approaches considered towards introduction the category of tensor in technical universities. Diverse alternative points of view in understanding tensors have been studied, as well as logical aspects of "tensor calculus architecture" cleared up. To our mind, a major challenge in tensors perception by undergraduates is a seamless transition from intuitively known "geometric vectors" to the core formalism of tensor calculus, i.e. to the first rank covariant and contravariant tensors which are basic terms in various high rank tensor forms.

Tensor analysis is a truly novel holistic insight on our world which

(C) V.I. Tikhonov, O.V. Tykhonova, 2018 
extends far beyond traditional distinct academic disciplines like physics, geometry, algebra etc. To understand complex tensors in geometrical interpretation as abstract spatial forms (or spaces) with unusual properties, our theoretical and empirical knowledge must be crucially rethought.

A cognitive point of view on matter is about to recognize that spatial structure of the world is not more than an individual model of a local physical environment which is perceived through human sensations and/or various instruments of observation. Thus, no "absolute space" exists, and none "objective coordinate" can be measured for "a point" or "vector" in either physical or geometrical orthonormal coordinate basis.

For this reason, with respect to tensors, the notorious question arises again. What comes first - matter or consciousness? In other words, what is primarily predetermined in a formal theory (physical experiment or its geometrical model, vector as object or vector space, tensor as geometrical image or tensor as an abstract algebraic coordinate form)? Without convincing and consistent logical doctrine, most academic tutorials on tensor analysis run the risk of being incomprehensible to the target audience.

Numerous attempts at constructive introduction to tensor analysis for students of technical universities, as well as obvious difficulties in understanding this discipline, once again reflected the known problem of axiomatic foundation of mathematics. This problem is usually associated with Cantor's set theory (also known as "naïve" set theory) introduced by German mathematician Georg Cantor in 1879 - 1883 [13].

The Cantor's set theory became a common axiomatic platform of classic functional analysis, and still, remains a fundamental universities course, though sharp discussions on this theory triggered since end of XIX century. Actually, the Cantor's "naive" set theory was designed as a "flat" (or singlelevel) formal grammar, where all the terms blend into one layer framework without any hierarchical subordination. Because of this, the "naive" set theory is experiencing internal contradictions. Philosophers and mathematicians of 19 - 20 centuries (Bertrand Russell, J. Von Neumann, Kurt Gödel, Paul Bernays, and others) discovered some logical problems caused due to the Cantor's set theory.

Bertrand Russell pointed at one of those problems in 1901 (aka "Russell's paradox" or Russell's antinomy [14]. In simple words, the "Russell's paradox" sounds as a rhetorical question ("is the set of all sets a subset of itself?") for which neither "yes" nor "no" are right answers.

The same paradox was earlier discovered by E. Zermelo (1899), but not published. At the end of the 1890s G. Cantor himself realized the cognitive issues of his set theory [15]. 
The nature of mentioned above logical issues and paradoxes is that average human brain needs some explicit hierarchy of terms and notions for clear perception a complex logical construction. In fact, the underlying background of any serious formal theory should rely on statistics retrieved from empirical data.

Therefore, the axiomatic foundation of a well-tailored theory is a particular delicate shell of empirical knowledge that accumulates and reflects previous experience. And if the axiomatic background of a theory is not enough clear and transparent for target audience, then the whole building of abstract theory will inevitably fall apart, no matter how beautiful and strict there seemed the theory itself.

An outstanding result in radical rethinking the overall framework of math theory and its axiomatic basis for a physical system was exhibited by the greatest physicist and mathematician of the 20th century J. Von Neumann in his work on math foundation of quantum mechanics ([15], 1927). To design a consistent math model of quantum mechanics system, Neumann had to additionally create a fundamentally new set theory based on a multilevel class hierarchy (von Neumann classes) as an alternative to Cantor's single-level set theory ([16], 1928).

The essence of Neumann's class theory is that various "sets" in the formal theory are divided into hierarchy classes, forming a multi-level logical scheme of concepts. New terms of theory are built as functions of subordinated argument-terms, and the rank of a new function-term is determined by the highest rank among the argument-terms. No recursive definitions of terms are admitted (such as "set of all sets" in Russell's paradox mentioned above).

By the middle of the 20th century, joint efforts of J. Von Neumann, K. Gödel and P. Bernays, resulted in a coherent axiomatic basis for the set theory (aka NGB axioms [17], 1954). Despite NGB axioms issued about 70 years ago, many conventional courses on functional analysis are still based on the "naive" set theory of G. Cantor ([18 - 20]).

The careful consideration of tensor methodology exhibited in university textbooks, indicates similar problems of cognitive nature, tied to non-classified "flat" categorization of different terms like "vector", "space", "tensor" etc. while introducing the terminology and formalisms of tensor calculus.

On this premise, the axiomatic foundation of tensor analysis is not accomplished yet, and therefore, new researches in this realm needed.

2. Related publications survey. Objectives. In this section, some common undergraduate tutorials on tensors issued in English within 2002 2016, are discussed. 


\section{Reference 1.}

Consider the introduction to tensors for students of physics and engineering in NASA Glenn research center, Ohio ([5], 2002). Have a look at pages $4 / 8$ and $5 / 9$ of the text (where the first number " 4 " means the page number in content table, while the second number " 8 " means related page in "pdf" file). The given text claims the following:

Page 4/8:

- Scalar: Tensor of rank $0 . \quad$ (magnitude only -1 component)

- Vector: Tensor of rank 1. (magnitude and one direction-3 components)

This terminology is suggestive. Why stop at rank 1 ? Why not go onto rank 2 , rank 3 , and so on.

- Dyad: Tensor of rank 2. (magnitude and two directions $-3^{2}=9$ components)

- Triad: Tensor of rank 3 . (magnitude and three directions $-3^{3}=27$ components)

- Etcetera...

\section{Page 5/9:}

In constructing a dyad product from two vectors, we form the term-by-term product of each of their individual components and add. If $\mathbf{U}$ and $\mathbf{V}$ are the two vectors under consideration, their dyad product is simply $\mathbf{U V}$. The dyad product $\mathbf{U V}$ is neither a dot nor a cross product. It is a distinct entity unto itself. If $\mathbf{U}=\mathrm{u}_{1} \mathbf{i}+\mathrm{u}_{2} \mathbf{j}+\mathrm{u}_{3} \mathbf{k}$ and $\mathbf{V}=\mathrm{v}_{1} \mathbf{i}+\mathrm{v}_{2} \mathbf{j}+\mathbf{v}_{3} \mathbf{k}$, then

$$
\mathbf{U V}=\mathrm{u}_{1} \mathrm{v}_{1} \mathbf{i} \mathbf{i}+\mathrm{u}_{1} \mathrm{v}_{2} \mathbf{i j}+\mathrm{u}_{1} \mathrm{v}_{3} \mathbf{i k}+\mathbf{u}_{2} \mathrm{v}_{1} \mathbf{j} \mathbf{i}+\cdots
$$

where $\mathbf{i}, \mathbf{j}$, and $\mathbf{k}$ are unit vectors in the usual sense and $\mathbf{i i}, \mathbf{i j}$, ik, etc. are unit dyads. In forming the product UV above, we simply "did what came naturally" (a favorite phrase of another of my professors!) from our knowledge of multiplying polynomials in elementary algebra. Notice that, by setting $u_{1} v_{1}=\mu_{11}, u_{1} v_{2}=\mu_{12}$, etc., this dyad can be rewritten as

$$
\mathbf{U V}=\mu_{11} \mathbf{i} \mathbf{i}+\mu_{12} \mathbf{i j}+\mu_{13} \mathbf{i k}+\mu_{21} \mathbf{j i}+\cdots
$$

Our remarks on the file [5] are the following.

1) Neither previously in the [5] nor in this text fragment of [5], the operations like ii, $\mathbf{i j}$ are defined. Suppose that unitary vector dyads ii, ij are scalar product multiplication, then must be: $\mathbf{i} \mathbf{i}=1, \mathbf{i j}=\cos \varphi(\mathbf{i}, \mathbf{j})$, etc. Because of that, the dyad $\mathbf{U V}$ must result in a single number.

2) The only formal definition in [5] for a tensor of rank $n$ is brought above (scalar -0 rank tensor, vector -1 rank tensor, dyad -2 rank tensor, triad -3 rank tensor etc.).

\section{Reference 2.}

The advanced version of [5] is "Foundations of Tensor Analysis for Students of Physics and Engineering" ([6]), which was published by the NASA Glenn research center in 2005.

Page 11/17 in [6] says: 
Multiplication.-As with vector multiplication, dyad multiplication may take one of several forms. The dyad products to be examined in the following sections are the inner product, the cross product, the product of a dyad and a scalar, and the direct product of two dyads.

Our remarks on the file [6] are the following.

1) Here introduced 4 special types of "dyad multiplications" (or "dyad products"): "inner product", "cross product", "product of dyad and scalar", "direct product of two dyads".

2) On the other hand, in mathematics also know very similar terms like "dot product", "scalar product", "inner product", "outer product of vectors", "vector product", "cross product", "dyad product", "scalar multiplication", "vector multiplication" etc. [21]. Such collection of similar terms seems to be over complicated and surplus for consistent definition the tensor entity.

3 ) In the content table of [6] the first mention of tensor is:

"Metric or Fundamental Tensor 24" I means, that "tensor" is not introduced formally as itself.

Reference 3.

"Tensors and their applications", Azad Inst. of technology (India), 2006 [7]. The Preface of this file on page /10 says: A quantity having magnitude only is called "scalar" and a quantity with magnitude and direction both, called "vector". But certain quantities are associated with two or more directions; such a quantity is called "tensor".

Next, page 6/23 of [7], section "Tensor algebra" says:

\subsection{INTRODUCTION}

A scalar (density, pressure, temperature, etc.) is a quantity whose specification (in any coordinate system) requires just one number. On the other hand, a vector (displacement, acceleration, force, etc.) is a quantity whose specification requires three numbers, namely its components with respect to some basis. Scalers and vectors are both special cases of a more general object called a tensor of order $n$ whose specification in any coordinate system requires $3^{n}$ numbers, called the components of tensor. In fact, scalars are tensors of order zero with $3^{\circ}=1$ component. Vectors are tensors of order one with $3^{1}=3$ components.

Page 7/24 says: 


\subsection{COVARIANT AND CONTRAVARIANT VECTORS (TENSOR OF RANK ONE)}

Let $\left(x^{1}, x^{2}, \ldots, x^{n}\right)$ or $x^{i}$ be coordinates of a point in $X$-coordinate system and $\left(\bar{x}^{1}, \bar{x}^{2}, \ldots, \bar{x}^{n}\right)$ or $\bar{x}^{i}$ be coordinates of the same point in the $Y$-coordinate system.

Let $A^{i}, i=1,2, \ldots, n$ (or $A^{1}, A^{2}, \ldots, A^{n}$ ) be $n$ functions of coordinates $x^{1}, x^{2}, \ldots, x^{n}$ in X-coordinate system. If the quantities $A^{i}$ are transformed to $\bar{A}^{i}$ in $Y$-coordinate system then according to the law of transformation

$$
\bar{A}^{i}=\frac{\partial \bar{x}^{i}}{\partial x^{j}} A^{j} \quad \text { or } \quad A^{j}=\frac{\partial x^{j}}{\partial \bar{x}^{i}} \bar{A}^{i}
$$

Then $A^{i}$ are called components of contravariant vector.

Similarly, covariant tensors of rank 1 are introduced in [7]. Our remark on the file [7] is: The two coordinate systems are used ( $X$ and $Y$ ) which are not explicitly bound herewith in declarations.

Reference 4.

"Introduction to vectors and tensors", Houston, Texas, 2010 [8].

Consider the terminology used in the file [8].

Page 15: "Intersection", "Sum", "Direct Sum of Subspaces", "Factor Spaces", "Inner Product Spaces", |Reciprocal Basis|.

Page 62/: "The factor space is also called a quotient space".

Page 76/ "Reciprocal Basis"(an analog of dual basis).

Page 158/166: "Spectral Decomposition for Hermitian Endomorphisms"

$$
\mathbf{U}=\left(\mathbf{A}^{*} \mathbf{A}\right)^{1 / 2}=\sum_{j=1}^{L} \lambda_{j}^{1 / 2} \mathbf{P}_{j}
$$

Section 31. Linear Functions, the Dual Space.

Page 203/211: vector in a space itself vs. covector in a dual space.

Section 32. The Second Dual Space, Canonical Isomorphisms.

Section 33. Multilinear Functions,

Tensors, page 218/226:

More specifically, a tensor of order $(p, q)$ on $\mathscr{V}$, where $p$ and $q$ are positive integers, is a $(p+q)$ linear function

$$
\underbrace{\mathscr{V} * \times \cdots \times \mathscr{V}}_{p \text { times }} * \underbrace{\mathscr{V} \times \cdots \times \mathscr{V}}_{q \text { times }} \rightarrow \mathscr{R}
$$

Our remark on the file [8] is: that was the first mention of "tensor" in the content table (it is straight multi rank tensor).

Tutorial reference 5 .

"Tensors: A guide for undergraduate students", 2013 [9].

Page 498/2: "A guide on tensors is proposed for undergraduate students in physics or engineering that ties directly to vector calculus in orthonormal coordinate systems. We show that once orthonormality is relaxed, a dual basis, 
together with the contravariant and covariant components, naturally emerges. Manipulating these components requires some skill that can be acquired more easily and quickly once a new notation is adopted".

Consider terminology: Page 499/3:

"Between any two vectors is defined a dot (or scalar) product, a commutative rule that associates a real number to each pair of vectors". "In the $3 \mathrm{D}$ space, a cross (or vector) product is defined".

Consider definition of tensor.

Page 500/4:

jectivity character we are after. Quantities with such a character and defined in an $\mathrm{N}$-dimensional space are called tensors and, more specifically, rth-rank tensors if they have $N^{r}$ components. Scalars, being single-component quantities, are then zero-rank tensors, while vectors, being $N$-component quantities, are first-rank tensors. Higher-rank tensors are...well, tensors!

Page 501/5:

Any vector can be expressed as a linear combination of the basis $\left\{\mathbf{e}_{a}\right\}$ so that

$$
\mathbf{A}=A^{a} \mathbf{e}_{a},
$$

which is equivalent to Eq. (3) except for the change in notation, where the components of the vector $\mathbf{A}$ in the basis $\left\{\mathbf{e}_{a}\right\}$ have been labelled with an upper index. However, if one asks if $A^{a}=\mathbf{e}_{a} \cdot \mathbf{A}$ or $\mathbf{A} \cdot \mathbf{B}=\sum_{a} A^{a} B^{a}$ still hold as in Eqs. (4) and (5), the answer is negative:

Our remarks on the file [9] are:

1) Quantities with such a character and defined in an N-dimensional space are called tensors and, more specifically, rth-rank tensors if they have $\mathrm{Nr}$ components.

Reference 6. "A gentle introduction to tensors", 2014 [10].

Page 1/2:

"Tensors and transformations are inseparable. To put it succinctly, tensors are geometrical objects over vector spaces, whose coordinates obey certain laws of transformation under change of basis. Vectors are simple and well-known examples of tensors, but there is much more to tensor theory than vectors". 
"I have used the coordinate approach to tensors, as opposed to the formal geometrical approach. Although this approach is a bit old fashioned, I still find it the easier to comprehend on first learning, especially if the learner is not a student of mathematics or physics".

Page 4/5:

Consider two bases $\left(\mathbf{e}_{1}, \mathbf{e}_{2}\right)$, which we will henceforth call the old basis, and $\left(\tilde{\mathbf{e}}_{1}, \tilde{\mathbf{e}}_{2}\right)$, which we will call the new basis. See, for example, Figure 1.4, in which we have brought the two bases to a common origin.

Since $\left(\mathbf{e}_{1}, \mathbf{e}_{2}\right)$ is a basis, each of the vectors $\left(\tilde{\mathbf{e}}_{1}, \tilde{\mathbf{e}}_{2}\right)$ can be uniquely expressed as a linear combination of $\left(\mathbf{e}_{1}, \mathbf{e}_{2}\right)$, similarly to (1.1):

$$
\begin{aligned}
\tilde{\mathbf{e}}_{1} & =\mathbf{e}_{1} S_{1}^{1}+\mathbf{e}_{2} S_{1}^{2} \\
\tilde{\mathbf{e}}_{2} & =\mathbf{e}_{1} S_{2}^{1}+\mathbf{e}_{2} S_{2}^{2}
\end{aligned}
$$

Page 14/15:

Vectors, covectors, and linear operators are all special cases of tensors. We will not attempt to define tensors in abstract terms, but settle for a coordinatebased definition, as follows.

A tensor of type (or valency) $(r, s)$ over an n-dimensional vector space is an object consisting of $n^{r+s}$ coordinates, denoted by the generic symbol $a_{j_{1} \ldots j_{s}}^{i_{1} \ldots i_{r}}$, and obeying the following change-of-basis transformation law:

$$
\tilde{a}_{j_{1} \ldots j_{s}}^{i_{1} \ldots i_{r}}=T_{k_{1}}^{i_{1}} \ldots T_{k_{r}}^{i_{r}} a_{m_{1} \ldots m_{s}}^{k_{1} \ldots k_{r}} S_{j_{1}}^{m_{1}} \ldots S_{j_{s}}^{m_{s}}
$$

Our remarks on the file [10] are the following.

1) Terminology: "old basis", "new basis", "vector", "covector", "linear operators", "tensor valency".

2) "We will not attempt to define tensors in abstract terms...".

Reference 7. "Tensor Analysis and Elementary Differential Geometry for Physicists and Engineers", Chapter 2 - Tensor Analysis, Berlin, 2014 [11].

Page 36/2 says: 
The definition of tensors is based on multilinear algebra with a multilinear map. We consider the real vector spaces $\mathbf{U}_{1}, \ldots, \mathbf{U}_{n}$ and their respective dual vector spaces $\mathbf{V}_{1}, \ldots, \mathbf{V}_{m}$. Each of their vector spaces belongs to the finite $N$-dimensional space $\mathbf{R}^{N}$, the image vector space $\mathbf{W}$, to the real space $\mathbf{R}$. A mixed tensor of type $(\boldsymbol{m}, \boldsymbol{n})$ can be defined as a multilinear functional T that maps an $(m+n)$ tuple of vectors of the vector spaces $\mathbf{U}$ and $\mathbf{V}$ into $\mathbf{W}$ (Fecko 2011) (Fig. 2.1):

$$
\begin{aligned}
& \mathbf{T}:\left(\mathbf{U}_{1} \times \cdots \times \mathbf{U}_{n}\right) \times\left(\mathbf{V}_{1} \times \cdots \times \mathbf{V}_{m}\right) \rightarrow \mathbf{W} \\
& \underbrace{\mathbf{R}^{N} \times \cdots \times \mathbf{R}^{N}}_{n \text { copies }} \times \underbrace{\mathbf{R}^{N} \times \cdots \times \mathbf{R}^{N}}_{m \text { copies }} \rightarrow \mathbf{R} \\
& \left(\mathbf{u}_{1}, \ldots, \mathbf{u}_{n} ; \mathbf{v}_{1}, \ldots, \mathbf{v}_{m}\right) \rightarrow \mathbf{T}\left(\mathbf{u}_{1}, \ldots, \mathbf{u}_{n} ; \mathbf{v}_{1}, \ldots, \mathbf{v}_{m}\right) \in \mathbf{R} .
\end{aligned}
$$

Our remarks on the file [11] following: This is a typical abstract definition of general case tensor form.

Reference 8. "Introduction to tensor calculus", Dep. of Physics \& Astronomy, Univ. College, London, 2016 [12].

Pages $(5-6) /(6-7)$ :

"Vectors are broadly geometric objects which are uniquely identified by their magnitude (length) and direction in a presumed underlying space". "At this early stage in these notes, we generically define "tensor" as an organized array of mathematical objects such as numbers or functions".

"Non-indexed (lower or upper case) bold face Latin letters (e.g. a, A) are used for vectors. The exception to this is the basis vectors where indexed bold face lower or upper case symbols are used".

"Non-indexed upper case bold face Latin letters (e.g. A, B) are used for tensors (i.e. of rank > 1)". "Indexed light face italic symbols (e.g. $a_{i}$ and $B_{j}^{k i}$ ) are used to denote tensors of rank $>0$ in their explicit tensor form (index notation). Such symbols may also be used to denote the components of these tensors".

Page 11/12: "A tensor is an array of mathematical objects (usually numbers or functions) which transforms according to certain rules under coordinates change. In a $d$-dimensional space, a tensor of rank- $n$ has $d^{n}$ components ...".

Page 13/14: "Each tensor index should conform ... either covariant or contravariant. For orthonormal Cartesian coordinate systems, the two variance types (i.e. covariant and contravariant) do not differ...".

Our remarks on the file [12] following. 1) Much attention paid to accurate and consistent notification of the terms. 2) Given definition of tensor is not explicit. 3) It is claimed, that in Cartesian coordinate system both two principal forms of tensor (covariant and contravariant) match up.

Page 30/31 says about the tensor multiplication. 
- This may also be called outer or exterior or direct or dyadic multiplication, although some of these names may be reserved for operations on vectors.

- On multiplying each component of a tensor of rank $r$ by each component of a tensor of rank $k$, both of dimension $m$, a tensor of rank $(r+k)$ with $m^{r+k}$ components is obtained where the variance type of each index (covariant or contravariant) is preserved, e.g.

$$
A_{i} B_{j}=C_{i j}
$$

Page 31/32: "Not every tensor can be synthesized as a product of lower rank tensors".

Page 32/33: "In general, the inner product is not commutative. When one or both of the tensors involved in the inner product are of rank $>1$ the order of the multiplicands does matter". Another fragment of this page is:

- As indicated before (see $\S 2.6 .4$ ), the dot product of two vectors is an example of the inner product of tensors, i.e. it is an inner product of two rank- 1 tensors to produce a rank-0 tensor:

$$
[\mathbf{a b}]_{i}^{j}=a_{i} b^{j} \quad \stackrel{\text { contraction }}{\longrightarrow} \quad \mathbf{a} \cdot \mathbf{b}=a_{i} b^{i}
$$

Our remarks on the file [12] (continued).

4) The tensor multiplication like $\otimes$ looks similar to matrix operations.

5) The statement on p.31/32 contradicts the general tensor definition.

6) The statement on p. 32/33 about the cumulativeness indicates the nonconsistence of given tensors introduction. Another statement provokes confusion around the terms "dot product", "inner product of tensors", "outer product", "scalar product" etc.

To our mind, too many identical terms are involved in such explanation. Also many special operations added into tensor algebra: contraction (p.31/32), permutation and quotient rule (p. 34/35), which is close to matrix algebra.

Analyzing the cited above tutorials on tensor calculus for students, we'll highlight the following cognitive aspects of tensor method.

1) There is no common definition of tensor among the specialists. Some of them introduce tensor as a special type of vector/covector multiplication (vectors understood as first rank tensors); other ones determine tensor through multiplication of vector spaces and dual vector spaces, taken from a common Euclidian space. 
2) The core issue of a dedicated introduction to tensor analysis is how to explicitly define the first rank covariant and contravariant tensors.

3) The "plane" thesaurus of basic terms in known textbooks on tensors (vector, space, tensor etc.) leads to logical contradictions noted above.

Objective of this work is outlining a cognitive tensor model of a system on the basis of Von Neumann classes of hierarchy.

\section{Outlines of tensor model based on Von Neumann classes}

In this section core outlines and definitions are presented for construction a holistic tensor model of a physical system based on hierarchical set theory by J. Von Neumann (aka Neumann's classes). The following methodological principles are proposed for a cognitive tensor modeling.

1) In a cognitive tensor model, the standard matrix algebra forms the operational basis (first of all, the well-known matrix multiplication rule, [22]). Therefore, no special operations and related cumbersome terms will be introduced for tensors (like Einstein's summation notation $x=x_{i} e^{i}$, dyad product uv , tensor multiplication $u \otimes v$, tensor components etc.).

2) In a cognitive tensor model, the set of empirical data in some physical units (e.g. power, energy) will form a core layer (zero-order Neumann's class) for construction subsequent tensor-related terms and objects (high-order Neumann's classes). Therefore, no predetermined spaces and their bases are needed more (like vector space, factor space, quotient space, reciprocal space, orthonormal basis, Cartesian basis, normal basis, affine basis etc.). Only two spatial categories (i.e. vector space $\mathbf{U}$ 离 and dual vector space $\mathbf{U}$ ) will be determined over initially given matrix $\mathbf{H}$ of empirical data.

3) In a cognitive tensor model, one more type of vector coordinates will be adopted (i.e. normal coordinates) in addition to conventional covariant and contravariant coordinates. This admits an accurate axiomatic definition of the first-rank covariant and contravariant tensors in terms of the first-order Neumann's class.

Based on declared above principles, we present a consistent axiomatic scheme for a cognitive tensor model of physical system. Let be given Hermitian matrix of empirical data measured on a physical system in abstract units of square magnitude $\left(\mu^{2}\right)$.

Axiom 1.

Let be given a nonsingular Hermitian matrix $\mathbf{H}_{\wp}$ of empirical data measured on a physical system $\wp$ in abstract units of square magnitude $\left(\mu^{2}\right)$. Matrix $\mathbf{H}_{\wp}$ considers be initial term of zero-order Neumann's class tied to system $\wp$. 


\section{Axiom 2.}

The Hermitian matrix $\mathbf{H}_{\wp}$ of empirical data measured on physical system $\wp$ in square magnitude units $\left(\mu^{2}\right)$, be mapped on a scalar product $\langle\overrightarrow{\mathbf{U}} \cdot \overrightarrow{\mathbf{U}}\rangle:=\mathbf{H}_{\wp}$ of an abstract vector rapper $\overrightarrow{\mathbf{U}}:=\left\{\overrightarrow{\mathbf{U}}_{1}, \overrightarrow{\mathbf{U}}_{2}, \mathbf{K}\right\}$ in linear magnitude units $\left(\mu^{1}\right)=(\mu)$. The scalar product $\langle\overrightarrow{\mathbf{U}} \cdot \overrightarrow{\mathbf{U}}\rangle$ and vector rapper $\overrightarrow{\mathbf{U}}$ consider be terms of the first-order Neumann's class.

Axiom 3.

3.1. The vector rapper $\overrightarrow{\mathbf{U}}$ in linear magnitude units $(\mu)$, which predefined by its scalar product $\langle\overrightarrow{\mathbf{U}} \cdot \overrightarrow{\mathbf{U}}\rangle$ retrieved from Hermitian matrix $\mathbf{H}_{\wp} \rightarrow\langle\overrightarrow{\mathbf{U}} \cdot \overrightarrow{\mathbf{U}}\rangle$ of empirical data measured on a physical system $\wp$ in square magnitude units $\left(\mu^{2}\right)$, determines a local in time Euclidian space $\overrightarrow{\mathbf{E}}\left(\mathbf{H}_{\wp}\right)$ tied to the system $\wp$.

3.2. The Euclidian space $\overrightarrow{\mathbf{E}}\left(\mathbf{H}_{\wp}\right)$ tied to the system $\wp$, is defined by the orthonormal coordinate basis $e^{\prime}:=\left\{\mathrm{e}_{1}, \mathrm{e}_{2}, \ldots\right\}$ in physical units of an abstract magnitude $(\mu)$ due to the presentation the vector rapper $\overrightarrow{\mathbf{U}}$ by the Hermitian matrix $\underline{\mathbf{U}}=+\sqrt{\mathbf{H}_{\wp}}$ of its normal coordinates in basis $\overrightarrow{\mathbf{e}}$.

Definition 1. Hermitian matrix $\underline{\mathbf{U}}=+\sqrt{\mathbf{H}_{\wp}}$ of the normal coordinates in basis 'e with physical units of an abstract magnitude $(\mu)$ we define as fundamental covariant (on rapper $\overrightarrow{\mathbf{U}}$ ) first rank tensor, which is determined in local Euclidian space $\overrightarrow{\mathbf{E}}\left(\mathbf{H}_{\wp}\right)$ [23].

Euclidian space $\overrightarrow{\mathbf{E}}\left(\mathbf{H}_{\wp}\right)$, basis $\dot{e}$ and fundamental tensor $\underline{\mathbf{U}}$ consider be terms of the first order Neumann's class. Along with the fundamental tensor $\underline{\mathbf{U}}$, which is determined above, some other fundamental first rank covariant tensors can be defined; all these tensors we call "fundamental" because of their common origin from Hermitian matrix $\mathbf{H}_{\wp}$ of empirical data tied to physical system $\wp$. Therefore, all the fundamental tensors are nonsingular (have their inverse forms), and each of a fundamental tensor uniquely defines the local Euclidian space $\overrightarrow{\mathbf{E}}\left(\mathbf{H}_{\wp}\right)$.

Definition 2. Let an arbitrary $\underline{\mathbf{Z}}$ be a fundamental (nonsingular) first-rank tensor, which is covariant on some vector rapper $\stackrel{\mathbf{Z}}{\mathbf{Z}} \mathbf{E}\left(\mathbf{H}_{\wp}\right)$ in a local 
Euclidian space $\mathbf{E}\left(\mathbf{H}_{\wp}\right)$. The object $\overline{\mathbf{Z}}:=\underline{\mathbf{Z}}^{-1}$ we define contravariant on $\mathbf{Z}$ first rank tensor in $\mathbf{E}\left(\mathbf{H}_{\wp}\right)$.

Definition 3. If $\mathbf{A}$ and $\mathbf{B}$ are fundamental first rank tensors, the matrix multiplication $\mathbf{C}:=\mathbf{A} \cdot \mathbf{B}^{*}$ we define as second-rank fundamental tensor in a local Euclidian space $\mathbf{E}\left(\mathbf{H}_{\wp}\right)$; the type of tensor $\mathbf{C}$ is defined by the types of multiplication members. Four types are possible hereby:

double covariant tensor $\mathbf{C}_{\mathbf{A B}}$, double contravariant tensor $\mathbf{C}^{\mathbf{A B}}$, covariant on $\mathbf{A}$ and contravariant on $\mathbf{B}$ tensor $\mathbf{C}_{\mathbf{A}}{ }^{\mathbf{B}}$, contravariant on $\mathbf{A}$ and covariant on B tensor $\mathbf{C}^{\mathbf{A}} \mathbf{B}$.

As matrix multiplication is not commutative operation, four other fundamental tensors of second rank can be constructed by $\mathbf{A}$ and $\mathbf{B}$ :

$$
\mathbf{C}:=\mathbf{B} \cdot \mathbf{A}^{*} \rightarrow \mathbf{C}_{\mathbf{B A}}, \mathbf{C}^{\mathrm{BA}}, \mathbf{C}_{\mathbf{B}}{ }^{\mathrm{A}}, \mathbf{C}^{\mathrm{B}}{ }_{\mathrm{A}} .
$$

All the fundamental tensors we declare as members of the first-order Neumann's class.

Definition 4. If an arbitrary nonsingular tuple of vectors $V^{\prime}$ is presented by the matrix $\underline{V}$ of normal coordinates in orthonormal basis $\dot{e}$ of Euclidian space $\mathbf{E}\left(\mathbf{H}_{\wp}\right)$, then matrix $\underline{V}$ we define as spawned covariant on $\dot{V}$ first rank tensor, and matrix $\bar{V}:=\underline{V}^{-1}-$ spawned contravariant on ${ }^{\prime}$ first rank tensor in $\mathbf{E}\left(\mathbf{H}_{\wp}\right)$. The multiplication of $r$ first-rank spawned tensors we define as $r$-rank spawned tensor. All the spawned tensors we declare as members of the second-order Neumann's class (concisely called "tensors").

\section{Conclusion.}

Tensor methodology is a powerful mathematical tool used in physics, system engineering and others realms. In technical universities, variety of approaches is used for tensors study. However, in known guides for undergraduate students tensors still remain about a mystery. Academic disciplines on tensors are not provided by the solid theoretical foundation, and most of the formal terms are commonly determined in a "flat" logical architecture inherent to Cantor set theory which suffers known paradoxes.

In present paper there applied hierarchical Neumann's classes to provide a holistic cognitive tensor model of a physical system. Three Neumann's classes are introduced: class 0 for empirical data on system relationships, class 1 for fundamental tensors, and class 2 for spawned tensors (concisely called "tensors").

This approach opens the way for further strict classification of tensors, in their connection with vector properties in elementary geometry and classical 
functional analysis in finite-dimensional Euclidean spaces. Eventually, the introduction to tensor algebra behind the Neumann's classes becomes more comprehensive and understandable by the target audience.

\section{References:}

1. Riemann, B. (1873), "On the Hypotheses which lie at the Bases of Geometry Nature", Vol. VIII, Nos. 183, 184, pp. 14-17, 36-37. - Available: https://www.emis.de/classics/ Riemann/WKCGeom.pdf.

2. Ricci-Curbastro, G. (1887), "Sulla derivazione covariante ad una forma quadratica differenziale", Rend. Acc. Lincei, Vol. 3 (4), pp. 15-18.

3. Levi-Civita, T. (1899), "Interpretazione gruppale degli integrali di un sistema canonico", Rend. Acc. Lincei, Vol. VII, pp. 235-238.

4. Einstein, A. (1913), "Entwurf einer verallgemeinerten Relativitätstheorie und einer Theorie der Gravitation", Zeitschrift für Mathematik und Physik, Vol. 62, pp. 225-261.

5. Kolecki, J.C. (2002), An introduction to tensors for students of physics and engineering [Electronic resource], NASA Glenn Research Center, Cleveland, Ohio, 29 P. - Available: https://ntrs.nasa.gov/archive/ nasa/casi.ntrs.nasa.gov/20020083040.pdf.

6. Kolecki, J.C. (2005), Foundations of tensor analysis for students of physics and engineering with an introduction to the theory of relativity [Electronic resource], NASA Glenn Research Center, Cleveland, Ohio, 84 P. - Available: https://ntrs.nasa.gov/archive/nasa/casi.ntrs. nasa.gov/20050175884.pdf.

7. Islam, N. (2006), "Tensors and their applications" [Electronic resource], New Age International (P) Ltd. Publishers, 245 p. - Available: https://www.researchgate.net/ publication/272047252_Tensors_and_TheirApplications.

8. Bowen, R.M. (2010), Introduction to vectors and tensors. Linear and multilinear algebra, [Electronic resource], 293 p. - Available: http://oaktrust.library.tamu.edu/bitstream/ handle/1969.1/2502/IntroductionToVectorsAnd TensorsVol1.pdf.

9. Battaglia, F. (2013), "Tensors: A guide for undergraduate students", American Association of Physics Teachers, No. 81 (7), pp. 498-511.

10. Porat, B. (2014), A Gentle Introduction to Tensors [Electronic resource], 86 p., Available: https://www.ese.wustl.edu/ nehorai/Porat_A_Gentle_Introduction_to_Tensors_2014.pdf.

11. Nguyen-Schäfer, H. (2014), "Tensor Analysis and Elementary Differential Geometry for Physicists and Engineers", Mathematical Engineering 21, Chapter 2. tensor analysis [Electronic resource], Springer Verlag Berlin Heidelberg, pp. 35-99. - Available: https://link.springer.com/content/pdf/bfm\%3A978-3-642-18617-2\%2F1.pdf.

12. Sochi, T. (2016), "Introduction to tensor calculus" [Electronic resource], Department of Physics \& Astronomy, University College London, 84 p. - Available: https://www.researchgate.net/publication/296707240_Introduction_to_Tensor_Calculus.

13. Cantor, G. (1883), "Ueber unendliche, lineare Punktmannichfaltigkeiten", Mathematische Annalen, No. 21 (4), pp. 545-591.

14. Russell, B. (1980), The Principles of Mathematics. Vol. 1 [Electronic resource], 798 p. Available: http://people.umass.edu/klement/pom/pom-portrait.pdf.

15. Neumann, Von J. (1927), "Mathematische Begründung der Quantenmechanik", Nachrichten von der Gesellschaft der Wissenschaften zu Göttingen, MathematischPhysikalische Klasse, pp. 1-57.

16. Neumann, Von J. (1928), Die Axiomatisierung der Mengenlehre, Mathematische Zeitschrift, No. 27, pp. 669-752.

17. Bernays, P. (1954), "A system of axiomatic set theory", Part VII, The journal of symbolic logic, Vol. 19, No. 2, pp. 81-96. - Available: https://core.ac.uk/download/pdf/85221775.pdf. 
18. Kreyszig, E. (1978), Introductory functional analysis with applications, University of Windsor, 688 p. - Available: http://www-personal.acfr.usyd.edu.au/spns/cdm/ resources/ Kreyszig\%20-\%20Introductory\%20Functional\%20Analysis\%20with\%20 Applications.pdf. 19. Teschl, G. (2010), "Topics in Real and Functional Analysis" [Electronic resource], American Mathematical Society Providence, Rhode, Island, 558 p. - Available: https://www.mat.univie.ac.at/ gerald/ftp/book-fa/fa.pdf.

20. Daners, D. (2017), Introduction to Functional Analysis [Electronic resource], School of Mathematics and Statistics University of Sydney, 117 p. - Available: http://www.maths.usyd.edu.au/u/ athomas/FunctionalAnalysis/daners-functional-analysis2017.pdf.

21. Engineering Math - Quick Reference [Electronic resource]. - Available: http://www.sharetechnote. com/html/Handbook_EngMath_Matrix_InnerProduct.html.

22. Gantmacher, F.R. (1960), The theory of matrices, Chelsea Publishing Company, N.Y., 276 p. - Available: https://www.maths.ed.ac.uk / v1 ranick/papers/gantmacher1.pdf.

23. Tikhonov, V. "A unified geometrical presentation of a quantum system experiment", Herald of the National Technical University "KhPI". Series of "Informatics and Modeling". 2018. - № 24 (1300). - P. 59-67.

The article is presented by doctor of technical sciences, professor, the head of "Radio and Television" depart. of the "Institute of Radio, Television and Information Security" of "O.S. Popov ONAT", Honored Worker of Science and Technology of Ukraine Gofiyzen O.V.

Received 12.11.2018

Tikhonov Victor, Doctor of technical science, associate professor Laureate of the Ukraine State Prize for Science and Technology, Professor of Communication Networks department

O.S. Popov Odessa National Academy of Telecommunications

Str. Kuznechna, 1, Odessa 65029, Ukraine

Tel.: 067-752-13-90

E-mail: victor.tykhonov@onat.edu.ua

Tykhonova Olena, Teacher of Communication Networks department O.S. Popov Odessa National Academy of Telecommunications

Str. Kuznechna, 1, Odessa 65029, Ukraine

Tel.: 097-490-56-18

E-mail: elena.tykhonova @onat.edu.ua 
УДК 514.8

Когнітивна тензорна модель системи на основі класів Фон Неймана / Тіхонов B.I., Тихонова О.В. // Вісник НТУ "ХПІ". Серія: Інформатика та моделювання. - Харків: НТУ "ХПІ". - 2018. - № 42 (1318). - С. 82 - 97.

$\mathrm{У}$ роботі розглядаються проблеми вивчення тензорного обчислення в технічних університетах. Для просування цілісного розуміння тензорів студентами, запропоновано когнітивну тензорну модель фізичної системи на основі ієрархічних класів Джона фон Неймана. Бібліогр.: 23 назв. Неймана.

Ключові слова: когнітивна тензорна модель; фізична система; класи Фон УДК 514.8

Когнитивная тензорная модель системы на основе классов Фон Неймана / Тихонов В.И., Тихонова Е.В. // Вестник НТУ "ХПИ". Серия: Информатика и моделирование. - Харьков: НТУ "ХПИ". - 2018. - № 42 (1318). - С. 82 97.

В статье рассматриваются проблемы изучения тензорного исчисления в технических университетах. Чтобы способствовать целостному пониманию тензоров студентами, предложена когнитивная тензорная модель физической системы на основе иерархических классов Джона фон Неймана. Библиогр.: 23 назв.

Ключевые слова: когнитивная тензорная модель; физическая система; классы Фон Неймана.

\section{UDC 514.8}

Cognitive tensor model of a system based on Von Neumann classes / Tikhonov V.I., Tykhonova O.V. // Herald of the National Technical University "KhPI". Series of "Informatics and Modeling". - Kharkov: NTU "KhPI". - 2018. - № 42 (1318). P. $82-97$.

The paper focuses issues of tensor calculus study in technical universities. To promote a holistic insight on tensors by undergraduates, there proposed a cognitive tensor model of a physical system on the base of hierarchical John Von Neumann classes. Refs.: 23 titles.

Keywords: cognitive tensor model; physical system; Neumann classes. 